\title{
Unsteady Mixed Convection Boundary Layer from a Circular Cylinder in a Micropolar Fluid
}

\author{
Anati Ali, ${ }^{1}$ Norsarahaida Amin, ${ }^{1}$ and Ioan Pop ${ }^{2}$ \\ ${ }^{1}$ Department of Mathematics, Faculty of Science, Universiti Teknologi Malaysia, Johor 81310, Malaysia \\ ${ }^{2}$ Faculty of Mathematics, University of Cluj, 400084 Cluj, Romania \\ Correspondence should be addressed to Anati Ali, anati@utm.my
}

Received 20 October 2009; Revised 7 June 2010; Accepted 5 July 2010

Academic Editor: Mostafa Barigou

Copyright ( $) 2010$ Anati Ali et al. This is an open access article distributed under the Creative Commons Attribution License, which permits unrestricted use, distribution, and reproduction in any medium, provided the original work is properly cited.

\begin{abstract}
Most industrial fluids such as polymers, liquid crystals, and colloids contain suspensions of rigid particles that undergo rotation. However, the classical Navier-Stokes theory normally associated with Newtonian fluids is inadequate to describe such fluids as it does not take into account the effects of these microstructures. In this paper, the unsteady mixed convection boundary layer flow of a micropolar fluid past an isothermal horizontal circular cylinder is numerically studied, where the unsteadiness is due to an impulsive motion of the free stream. Both the assisting (heated cylinder) and opposing cases (cooled cylinder) are considered. Thus, both small and large time solutions as well as the occurrence of flow separation, followed by the flow reversal are studied. The flow along the entire surface of a cylinder is solved numerically using the Keller-box scheme. The obtained results are compared with the ones from the open literature, and it is shown that the agreement is very good.
\end{abstract}

\section{Introduction}

The unsteady nature of a wide range of fluid flows of practical importance has received considerable attention in recent years. In many applications, the ideal flow environment around a device is nominally steady, but undesirable unsteady effects arise either due to self-induced motion of the body, or due to the fluctuations or nonuniformities in the surrounding fluid. On the other hand, some devices are required to execute time-dependent motion in order to perform their basis functions (McCroskey [1]). The fluid dynamic aspects of some of these problems can normally be approximated by small departures from steady behavior, and some cannot. In general, unsteady viscous phenomena play an important role in the reentry of space vehicles. Such phenomena, as for example, the growth of separated bubble or the displacement of the point of separation also appear in the study of flow around a helicopter blade or stalling of airfoil. The rotor blades of helicopters in forward flight translate more nearly in the plane of rotation than axially. This introduces still another type of unsteadiness, because relative to the individual blade elements, the local approaching air stream varies periodically with largeamplitude fluctuations in its magnitude, yaw angle, and chordwise incidence (McCroskey [1]). Fluid motion in the human blood vessels is also unsteady and appears to involve regions of reversed flow. Unsteady viscous flows have been studied quite extensively and all the characteristic features of unsteady effects are now more or less familiar to fluid mechanicists. Stewartson [2], Stuart [3], McCroskey [1], Riley [4, 5], Telionis [6], and Pop et al. [7] have concisely reviewed the main ideas and important contributions on the topic.

The classical problem of unsteady forced convection boundary layer flow of a viscous and incompressible fluid past a circular cylinder has been considered by many authors, such as Collins and Dennis $[8,9]$, Telionis and Tsahalis [10], Bar-Lev and Yang [11], Patel [12], Takao [13], Cebeci $[14,15]$, Ingham [16], and Nam [17]. By using the Keller-box method in three dimensional grid with zig-zag differencing, Cebeci $[14,15]$ solved the problems of unsteady forced convection flow and heat transfer reversal past a circular cylinder. Further, Ali et al. [18] extended the problem considered by Cebeci [14] to the unsteady forced convection 
boundary layer flow past an isothermal circular cylinder immersed in a micropolar fluid. It is worth mentioning that the problem of steady mixed convection boundary layer flow past an isothermal circular cylinder in a Newtonian fluid, when the an impulsive motion of the free stream. Both the assisting (heated cylinder) and opposing (cooled cylinder) cases has been considered by Merkin [19]. The horizontal cylinder is placed in a stream flowing vertically upwards. $\mathrm{He}$ found that heating the cylinder would delay separation and if the cylinder is warm enough, there will be no separation at all. On the other hand, cooling the cylinder brings the separation point nearer to the lower stagnation point and for a sufficiently cold cylinder; there will not be a boundary layer on the cylinder. Ingham and Merkin [20] extended this problem to the case of unsteady mixed convection boundary layer. The numerical solutions of the governing equations were presented for various station along the cylinder. In micropolar fluid, the steady mixed convection boundary layer flow over an isothermal horizontal cylinder has been studied by Nazar et al. [21]. The Keller-box method was applied to solve the problem for both cases of heated (assisting flow) and cooled (opposing flow) cylinder. The calculation was carried up to the point of separation of the boundary layer from the surface of the cylinder. It is important to notice at this end that the micropolar model of fluid flow has attracted considerable attention from researchers since the formulation of the model by Eringen [22]. Eringen's micropolar model includes the classical Navier-Stokes equations as a special case, but can cover, both in theory and applications, many more phenomena than the classical model. Examples of industrially relevant flows that can be studied using the theory of micropolar fluids include the flow of low concentration suspensions, liquid crystals, blood, porous media, lubrication, turbulent shear flow, and so forth. Extensive reviews of the theory and applications can be found in the papers by Ariman et al. $[23,24]$ and in the books by Eringen [25] and Łukaszewicz [26]. Some experimental determination of material micropolar fluid has been done by Kolpashchikov et al. [27].

In this paper, we extend the work by Nazar et al. [21] on the steady mixed convection boundary layer flow past an isothermal horizontal cylinder placed in a micropolar fluid to the unsteady case by using the Keller-box method described in Ali et al. [18]. The flow beyond the point of separation has also been described. The numerical results in the form of skin friction coefficient, Nusselt number and separation time are presented for both cases of assisting flow (heated cylinder) and opposing flow (cooled cylinder) for some values of micropolar material parameter and Prandtl numbers. The papers by Kumari and Nath [28] and Agarwal et al. [29] describe the unsteady flow of a micropolar fluid but only at a stagnation point. To our knowledge such very detailed solutions have not previously been presented for the present problem.

\section{Governing Equations}

We consider a horizontal circular cylinder of radius $a$ surrounded by a micropolar fluid at rest and at the uniform
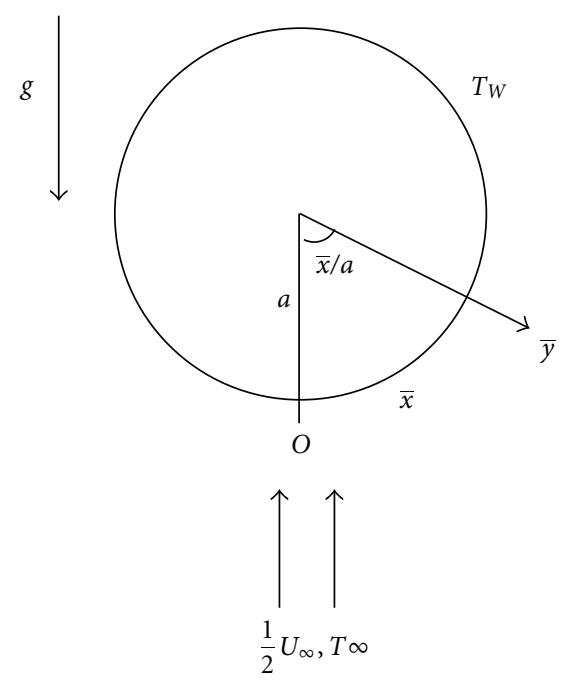

Figure 1: The physical model and coordinate system.

temperature $T_{\infty}$, which is suddenly changed at time $\bar{t}=0$ to $T_{w}$, where $T_{w}>T_{\infty}$ (heated cylinder) and $T_{w}<T_{\infty}$ (cooled cylinder), respectively. The physical geometry and coordinate system are illustrated in Figure 1.

Following Merkin [19], we assume that at the same time a uniform stream $(1 / 2) U_{\infty}$ at large distance from the cylinder is impulsively started and flows vertically upwards. Comparing our problem with Ingham and Merkin [20], another extra equation should be considered due to the presence of micropolar fluid which is the angular momentum equation. The dimensional governing equations for the problem considered in this study consist of the continuity, momentum, energy and microrotation equations as follows:

$$
\begin{gathered}
\frac{\partial \bar{u}}{\partial \bar{x}}+\frac{\partial \bar{v}}{\partial \bar{y}}=0 \\
\frac{\partial \bar{u}}{\partial \bar{t}}+\bar{u} \frac{\partial \bar{u}}{\partial \bar{x}}+\bar{v} \frac{\partial \bar{u}}{\partial \bar{y}}=\bar{u}_{e} \frac{d \bar{u}_{e}}{d \bar{x}}+\left(\frac{\mu+\kappa}{\rho}\right) \frac{\partial^{2} \bar{u}}{\partial \bar{y}^{2}} \\
+\frac{\kappa}{\rho} \frac{\partial \bar{N}}{\partial \bar{y}}+g \beta\left(\bar{T}-T_{\infty}\right) \sin \left(\frac{\bar{x}}{a}\right) \\
\frac{\partial \bar{T}}{\partial \bar{t}}+\bar{u} \frac{\partial \bar{T}}{\partial \bar{x}}+\bar{v} \frac{\partial \bar{T}}{\partial \bar{y}}=\frac{\nu}{\operatorname{Pr} \frac{\partial^{2} \bar{T}}{\partial \bar{y}^{2}}} \\
\rho j\left(\frac{\partial \bar{N}}{\partial \bar{t}}+\bar{u} \frac{\partial \bar{N}}{\partial \bar{x}}+\bar{v} \frac{\partial \bar{N}}{\partial \bar{y}}\right)=\left(\mu+\frac{\kappa}{2}\right) j \frac{\partial^{2} \bar{N}}{\partial \bar{y}^{2}}-\kappa\left(2 \bar{N}+\frac{\partial \bar{u}}{\partial \bar{y}}\right),
\end{gathered}
$$

which are subject to the initial and boundary conditions

$$
\begin{array}{ll}
\bar{t}<0: & \bar{u}=\bar{v}=\bar{N}=0, \quad \bar{T}=T_{\infty} \quad \text { for any } \bar{x}, \bar{y}, \\
\bar{t} \geq 0: \quad \bar{u}=\bar{v}=0, \quad \bar{N}=-n \frac{\partial \bar{u}}{\partial \bar{y}}, \quad \bar{T}=T_{w} \quad \text { at } \bar{y}=0, \\
\\
\bar{u}=\bar{u}_{e}(\bar{x}), \quad \bar{N}=0, \quad \bar{T}=T_{\infty} \quad \text { as } \bar{y} \longrightarrow \infty .
\end{array}
$$


TABLe 1: The separation times along the cylinder surface when $\operatorname{Pr}=1$ and $\alpha=-3$ (opposing flow).

\begin{tabular}{|c|c|c|c|c|c|c|c|}
\hline \multirow{2}{*}{$x$} & \multirow{2}{*}{$K=0$} & \multicolumn{2}{|c|}{$K=1$} & \multicolumn{2}{|c|}{$K=2$} & \multicolumn{2}{|c|}{$K=3$} \\
\hline & & $n=0$ & $n=1 / 2$ & $n=0$ & $n=1 / 2$ & $n=0$ & $n=1 / 2$ \\
\hline $180^{\circ}$ & 0.2194 & 0.2410 & 0.2463 & 0.2489 & 0.2627 & 0.2530 & 0.2746 \\
\hline $171^{\circ}$ & 0.2205 & 0.2424 & 0.2475 & 0.2501 & 0.2641 & 0.2542 & 0.2762 \\
\hline $162^{\circ}$ & 0.2234 & 0.2456 & 0.2510 & 0.2538 & 0.2683 & 0.2583 & 0.2809 \\
\hline $153^{\circ}$ & 0.2284 & 0.2513 & 0.2571 & 0.2602 & 0.2754 & 0.2651 & 0.2888 \\
\hline $144^{\circ}$ & 0.2356 & 0.2599 & 0.2660 & 0.2697 & 0.2858 & 0.2752 & 0.2984 \\
\hline $135^{\circ}$ & 0.2439 & 0.2715 & 0.2782 & 0.2825 & 0.2977 & 0.2874 & 0.3133 \\
\hline $126^{\circ}$ & 0.2557 & 0.2852 & 0.2920 & 0.2970 & 0.3154 & 0.3045 & 0.3313 \\
\hline $117^{\circ}$ & 0.2706 & 0.3033 & 0.3112 & 0.3179 & 0.3361 & 0.3258 & 0.3554 \\
\hline $108^{\circ}$ & 0.2875 & 0.3254 & 0.3337 & 0.3418 & 0.3638 & 0.3530 & 0.3847 \\
\hline $99^{\circ}$ & 0.3090 & 0.3527 & 0.3627 & 0.3725 & 0.3959 & 0.3866 & 0.4215 \\
\hline $90^{\circ}$ & 0.3337 & 0.3855 & 0.3961 & 0.4102 & 0.4363 & 0.4286 & 0.4671 \\
\hline $81^{\circ}$ & 0.3640 & 0.4252 & 0.4373 & 0.4564 & 0.4857 & 0.4809 & 0.5233 \\
\hline $72^{\circ}$ & 0.3978 & 0.4726 & 0.4866 & 0.5126 & 0.5456 & 0.5455 & 0.5922 \\
\hline $63^{\circ}$ & 0.4372 & 0.5285 & 0.5447 & 0.5804 & 0.6173 & 0.6249 & 0.6762 \\
\hline $54^{\circ}$ & 0.4812 & 0.5933 & 0.6120 & 0.6609 & 0.7022 & 0.7211 & 0.7771 \\
\hline $45^{\circ}$ & 0.5289 & 0.6660 & 0.6876 & 0.7539 & 0.7999 & 0.8346 & 0.8958 \\
\hline $36^{\circ}$ & 0.5780 & 0.7442 & 0.7689 & 0.8568 & 0.9079 & 0.9633 & 1.0304 \\
\hline $27^{\circ}$ & 0.6250 & 0.8221 & 0.8500 & 0.9625 & 1.0192 & 1.0986 & 1.1717 \\
\hline $18^{\circ}$ & 0.6651 & 0.8908 & 0.9217 & 1.0589 & 1.1201 & 1.2245 & 1.3037 \\
\hline $9^{\circ}$ & 0.6916 & 0.9388 & 0.9726 & 1.1276 & 1.1925 & 1.3163 & 1.4013 \\
\hline $0^{\circ}$ & 0.7012 & 0.9562 & 0.9901 & 1.1525 & 1.2190 & 1.3500 & 1.4374 \\
\hline
\end{tabular}

where $\bar{u}_{e}(\bar{x})=U_{\infty} \sin (\bar{x} / a)$ is the free stream velocity given by Merkin [19].

We have assumed in (4) that the spin-gradient viscosity $\gamma$ is defined as $\gamma=(\mu+\kappa / 2) j$, see Ahmadi [30]. Further, it should be pointed out that $n$ is a constant such that $0 \leq n \leq 1$. The case $n=0$, called strong concentration by Guram and Smith [31], indicating $\bar{N}=0$ near the wall, represents concentrated particle flows in which the microelements close to the wall surface are unable to rotate (Jena and Mathur [32]). The case $n=1 / 2$ indicates the vanishing of antisymmetrical part of the stress tensor and denotes weak concentration (Ahmadi [30]). The case $n=1$ is used for the modeling of turbulent boundary layer flows. In order to reduce the length of this paper, the results are presented only for the cases $n=0$ (strong concentration) and $n=1 / 2$ (weak concentration).

In order to obtain (1)-(4) in nondimensional form, we introduce the following non-dimensional variables:

$$
\begin{gathered}
x=\frac{\bar{x}}{a}, \quad y=\operatorname{Re}^{1 / 2}\left(\frac{\bar{y}}{a}\right), \quad t=\left(\frac{U_{\infty}}{a}\right) \bar{t}, \\
u=\frac{\bar{u}}{U_{\infty}}, \quad v=\operatorname{Re}^{1 / 2}\left(\frac{\bar{v}}{U_{\infty}}\right), \quad u_{e}=\frac{\bar{u}_{e}}{U_{\infty}}, \\
N=\operatorname{Re}^{-1 / 2}\left(\frac{a \bar{N}}{U_{\infty}}\right), \quad T=\frac{\left(\bar{T}-T_{\infty}\right)}{\left(T_{w}-T_{\infty}\right)} .
\end{gathered}
$$

Substituting these non-dimensional variables into (1)(4), we obtain

$$
\begin{aligned}
\frac{\partial u}{\partial x}+\frac{\partial v}{\partial y}= & 0 \\
\frac{\partial u}{\partial t}+u \frac{\partial u}{\partial x}+v \frac{\partial u}{\partial y}= & -u_{e} \frac{d u_{e}}{d x}+(1+K) \frac{\partial^{2} u}{\partial y^{2}} \\
& +K \frac{\partial N}{\partial y}+\alpha T \sin x \\
\frac{\partial T}{\partial t}+u \frac{\partial T}{\partial x}+v \frac{\partial T}{\partial y}= & \frac{1}{\operatorname{Pr}} \frac{\partial^{2} T}{\partial y^{2}} \\
\frac{\partial N}{\partial t}+u \frac{\partial N}{\partial x}+v \frac{\partial N}{\partial y}= & \left(1+\frac{K}{2}\right) \frac{\partial^{2} N}{\partial y^{2}}-K\left(2 N+\frac{\partial u}{\partial y}\right)
\end{aligned}
$$

where $\alpha=\mathrm{Gr} / \mathrm{Re}^{2}$ is the mixed convection parameter and $\mathrm{Gr}=g \beta\left(T_{w}-T_{\infty}\right) a^{3} / v^{2}$ is the Grashof number. It should be noted that the flow near the heated body $\left(T_{w}>T_{\infty}\right)$ leads to a positive value of the mixed convection parameter $\alpha$ $(\alpha>0)$ and the resulting buoyancy force aids the convective motion. This is called the assisting flow. On the other hand, $T_{w}<T_{\infty}$ (cooled body), leads to a negative value of the mixed convection parameter $\alpha(<0)$. This cooled body case is called 
the opposing flow since the buoyancy forced would oppose the flow. The initial and boundary conditions (5) become

$$
\begin{aligned}
t<0: & u=v=N=T=0 \quad \text { for any } x, y, \\
t \geq 0: & u=v=0, \quad N=-n \frac{\partial u}{\partial y}, \quad T=1 \quad \text { at } y=0, \\
& u=u_{e}(x), \quad N=0, \quad T=0 \quad \text { as } y \longrightarrow \infty .
\end{aligned}
$$

Equations (7) is a system of four equations with two velocity components $u$ and $v$. In two-dimensional flow, these velocity components at a given point can be expressed by the partial derivatives of the stream function $\psi$ using the following relations:

$$
u=\frac{\partial \psi}{\partial y}, \quad v=-\frac{\partial \psi}{\partial x} .
$$

Definition of (9) enables us to relate the velocity $u$ and $v$ to only one variable $\psi$. The continuity equation is automatically satisfied which reduce the system of (7) to a system of only three equations as follows:

$$
\begin{aligned}
\frac{\partial^{2} \psi}{\partial y \partial t}+\frac{\partial \psi}{\partial y} \frac{\partial^{2} \psi}{\partial y \partial x}-\frac{\partial \psi}{\partial x} \frac{\partial^{2} \psi}{\partial y^{2}}= & u_{e} \frac{d u_{e}}{d x}+(1+K) \frac{\partial^{3} \psi}{\partial y^{3}} \\
& +K \frac{\partial N}{\partial y}+\alpha T \sin x \\
\frac{\partial T}{\partial t}+\frac{\partial \psi}{\partial y} \frac{\partial T}{\partial x}-\frac{\partial \psi}{\partial x} \frac{\partial T}{\partial y}= & \frac{1}{\operatorname{Pr} \frac{\partial^{2} T}{\partial y^{2}}} \\
\frac{\partial N}{\partial t}+\frac{\partial \psi}{\partial y} \frac{\partial N}{\partial x}-\frac{\partial \psi}{\partial x} \frac{\partial N}{\partial y}= & \left(1+\frac{K}{2}\right) \frac{\partial^{2} N}{\partial y^{2}} \\
& -K\left(2 N+\frac{\partial^{2} \psi}{\partial y^{2}}\right)
\end{aligned}
$$

while the initial and boundary conditions (8) become

$$
\begin{aligned}
t<0: & \frac{\partial \psi}{\partial x}=\frac{\partial \psi}{\partial y}=N=T=0 \quad \text { for any } x, y \\
t \geq 0: & \frac{\partial \psi}{\partial x}=\frac{\partial \psi}{\partial y}=0, \quad N=-n \frac{\partial^{2} \psi}{\partial y^{2}}, \quad T=1 \quad \text { at } y=0 \\
& \frac{\partial \psi}{\partial y}=u_{e}(x), \quad N=0, \quad T=0 \quad \text { as } y \rightarrow \infty
\end{aligned}
$$

Immediately after the flow starts, a boundary layer is developed around the cylinder with the thickness of $O(v t)^{1 / 2}$. This argument is adopted according to the Rayleigh's solution where the boundary layer starts off with zero thickness at $t=0$ and grows with the square root of time (Telionis [6]). This suggests that, to obtain a solution for small time, (10) subject to the initial and boundary conditions (11) are transformed using the following similarity variables

$$
\begin{gathered}
\psi=t^{1 / 2} u_{e}(x) f(x, \eta, t), \quad N=t^{-1 / 2} u_{e}(x) h(x, \eta, t), \\
\eta=\frac{y}{t}^{1 / 2} .
\end{gathered}
$$

These variables are applied only for small time case $\left(t \leq t^{*}\right)$ where we choose $t^{*}=1$. Following Cebeci $[14,15]$, the following similarity variables are defined:

$$
\psi=u_{e} F(x, Y, t), \quad N=u_{e} H(x, Y, t), \quad Y=y,
$$

for large time $\left(t>t^{*}\right)$ case in order to avoid the effect of the singular initial condition on the numerical computation. Substituting (12) into (10) subject to (11), we obtain the following system of equations for small time $\left(t \leq t^{*}\right)$ case

$$
\begin{gathered}
(1+K) \frac{\partial^{3} f}{\partial \eta^{3}}+\frac{\eta}{2} \frac{\partial^{2} f}{\partial \eta^{2}}+t \frac{d u_{e}}{d x}\left[1-\left(\frac{\partial f}{\partial \eta}\right)^{2}+f \frac{\partial^{2} f}{\partial \eta^{2}}\right]+K \frac{\partial h}{\partial \eta} \\
=t \frac{\partial^{2} f}{\partial \eta \partial t}+t u_{e}\left(\frac{\partial f}{\partial \eta} \frac{\partial^{2} f}{\partial \eta \partial x}-\frac{\partial f}{\partial x} \frac{\partial^{2} f}{\partial \eta^{2}}\right)-t \alpha \frac{\sin x}{u_{e}} s, \\
\frac{\partial^{2} s}{\partial \eta^{2}}+\frac{\operatorname{Pr} \eta}{2} \frac{\partial s}{\partial \eta}+\operatorname{Pr} t \frac{d u_{e}}{d x} f \frac{\partial s}{\partial \eta} \\
=\operatorname{Pr} t\left[\frac{\partial s}{\partial t}+u_{e}\left(\frac{\partial f}{\partial \eta} \frac{\partial s}{\partial x}-\frac{\partial s}{\partial \eta} \frac{\partial f}{\partial x}\right)\right], \\
\left(1+\frac{K}{2}\right) \frac{\partial^{2} h}{\partial \eta^{2}}+\frac{\eta}{2} \frac{\partial h}{\partial \eta}+\frac{1}{2} h+t \frac{d u_{e}}{d x}\left(f \frac{\partial h}{\partial \eta}-h \frac{\partial f}{\partial \eta}\right) \\
=t \frac{\partial h}{\partial t}+t u_{e}\left(\frac{\partial f}{\partial \eta} \frac{\partial h}{\partial x}-\frac{\partial f}{\partial x} \frac{\partial h}{\partial \eta}\right)+t K\left(2 h+\frac{\partial^{2} f}{\partial \eta^{2}}\right),
\end{gathered}
$$

subject to the initial and boundary conditions

$$
\begin{array}{cl}
t<0: & f=\frac{\partial f}{\partial \eta}=h=s=0 \quad \text { for any } x, \eta \\
t \geq 0: & f=\frac{\partial f}{\partial \eta}=0, \quad h=-n \frac{\partial^{2} f}{\partial \eta^{2}}, \quad s=1 \quad \text { at } \eta=0 \\
& \frac{\partial f}{\partial \eta}=1, \quad h=0, \quad s=0 \quad \text { as } \eta \longrightarrow \infty .
\end{array}
$$

For large time $\left(t>t^{*}\right)$ case, the similarity variables (13) are substituted into (10) which gives

$$
\begin{gathered}
(1+K) \frac{\partial^{3} F}{\partial Y^{3}}+\frac{d u_{e}}{d x}\left[1+F \frac{\partial^{2} F}{\partial Y^{2}}-\left(\frac{\partial F}{\partial Y}\right)^{2}\right]+K \frac{\partial H}{\partial Y} \\
=\frac{\partial^{2} F}{\partial Y \partial t}+u_{e}\left(\frac{\partial F}{\partial Y} \frac{\partial^{2} F}{\partial Y \partial x}-\frac{\partial F}{\partial x} \frac{\partial^{2} F}{\partial Y^{2}}\right), \\
\frac{\partial^{2} S}{\partial Y^{2}}+\operatorname{Pr} \frac{d u_{e}}{d x} F \frac{\partial S}{\partial Y}=\operatorname{Pr}\left[\frac{\partial S}{\partial t}+u_{e}\left(\frac{\partial F}{\partial Y} \frac{\partial S}{\partial x}-\frac{\partial S}{\partial Y} \frac{\partial F}{\partial x}\right)\right], \\
\left(1+\frac{K}{2}\right) \frac{\partial^{2} H}{\partial Y^{2}}+\frac{d u_{e}}{d x}\left(F \frac{\partial H}{\partial Y}-H \frac{\partial F}{\partial Y}\right)-K\left(2 H+\frac{\partial^{2} F}{\partial Y^{2}}\right) \\
=\frac{\partial H}{\partial t}+u_{e}\left(\frac{\partial F}{\partial Y} \frac{\partial H}{\partial x}-\frac{\partial H}{\partial Y} \frac{\partial F}{\partial x}\right) .
\end{gathered}
$$


TABLE 2: The separation times along the cylinder surface when $\operatorname{Pr}=7$ and $\alpha=-3$.

\begin{tabular}{|c|c|c|c|c|c|c|c|}
\hline \multirow{2}{*}{$x$} & \multirow{2}{*}{$K=0$} & \multicolumn{2}{|c|}{$K=1$} & \multicolumn{2}{|c|}{$K=2$} & \multicolumn{2}{|c|}{$K=3$} \\
\hline & & $n=0$ & $n=1 / 2$ & $n=0$ & $n=1 / 2$ & $n=0$ & $n=1 / 2$ \\
\hline $180^{\circ}$ & 0.3153 & 0.3447 & 0.3558 & 0.3513 & 0.3779 & 0.3542 & 0.3929 \\
\hline $171^{\circ}$ & 0.3174 & 0.3470 & 0.3583 & 0.3538 & 0.3809 & 0.3568 & 0.3958 \\
\hline $162^{\circ}$ & 0.3234 & 0.3541 & 0.3660 & 0.3615 & 0.3890 & 0.3651 & 0.4049 \\
\hline $153^{\circ}$ & 0.3339 & 0.3667 & 0.3794 & 0.3752 & 0.4035 & 0.3796 & 0.4212 \\
\hline $144^{\circ}$ & 0.3478 & 0.3844 & 0.3975 & 0.3940 & 0.4249 & 0.3994 & 0.4439 \\
\hline $135^{\circ}$ & 0.3687 & 0.4099 & 0.4238 & 0.4214 & 0.4554 & 0.4287 & 0.4768 \\
\hline $126^{\circ}$ & 0.3946 & 0.4433 & 0.4594 & 0.4587 & 0.4961 & 0.4691 & 0.5218 \\
\hline $117^{\circ}$ & 0.4294 & 0.4884 & 0.5073 & 0.5093 & 0.5513 & 0.5245 & 0.5832 \\
\hline $108^{\circ}$ & 0.4745 & 0.5488 & 0.5702 & 0.5783 & 0.6261 & 0.6016 & 0.6675 \\
\hline $99^{\circ}$ & 0.5330 & 0.6300 & 0.6611 & 0.6741 & 0.7286 & 0.7111 & 0.7848 \\
\hline $90^{\circ}$ & 0.6092 & 0.7410 & 0.7652 & 0.8099 & 0.8714 & 0.8709 & 0.9520 \\
\hline $81^{\circ}$ & 0.7091 & 0.8954 & 0.9316 & 1.0072 & 1.0741 & 1.1106 & 1.1943 \\
\hline $72^{\circ}$ & 0.8408 & 1.1133 & 1.1448 & 1.2971 & 1.3641 & 1.4735 & 1.5510 \\
\hline $63^{\circ}$ & 1.0156 & 1.4222 & 1.4613 & 1.7253 & 1.7858 & 2.0198 & 2.0813 \\
\hline $54^{\circ}$ & 1.2456 & 1.8649 & - & - & - & - & - \\
\hline $45^{\circ}$ & 1.5474 & - & - & - & - & - & - \\
\hline $36^{\circ}$ & 1.9405 & - & - & - & - & - & - \\
\hline $27^{\circ}$ & 2.4357 & - & - & - & - & - & - \\
\hline
\end{tabular}

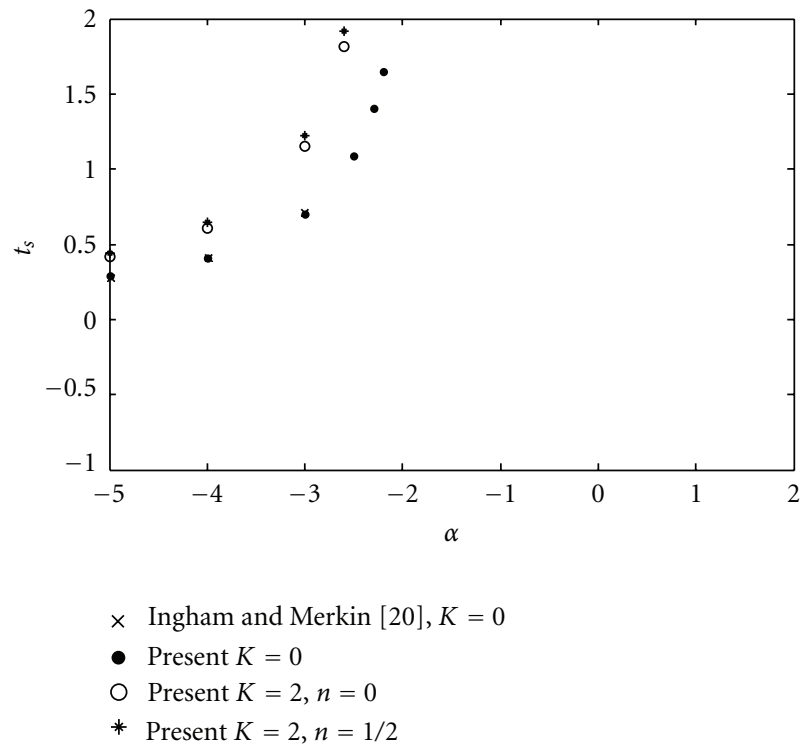

(a)

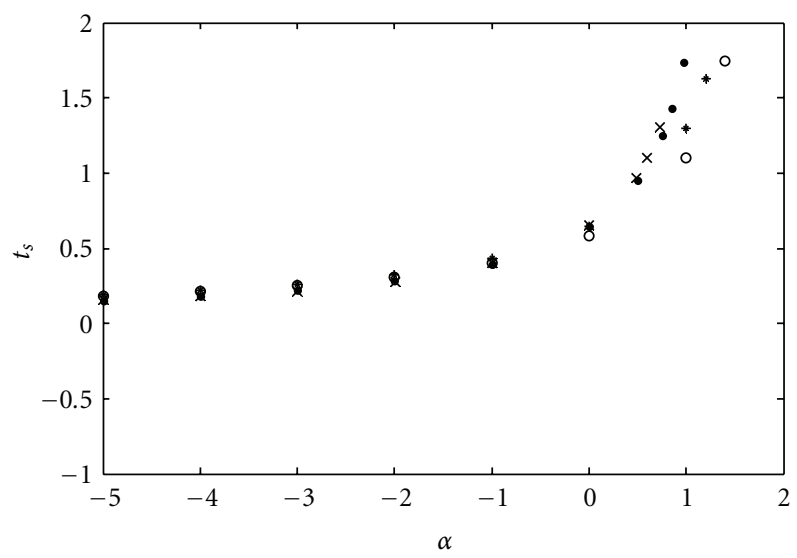

$\times$ Ingham and Merkin [20], $K=0$

- Present $K=0$

O Present $K=2, n=0$

* Present $K=2, n=1 / 2$

(b)

FIGURE 2: Variation of the separation times with $\alpha$ near: (a) forward and (b) rear stagnation points when $\operatorname{Pr}=1$ for $K=0,2$ and $n=0,1 / 2$.

Equations (16) are subjected to boundary conditions

$$
\begin{gathered}
F=\frac{\partial F}{\partial Y}=0, \quad H=-n \frac{\partial^{2} F}{\partial Y^{2}}, \quad S=1 \quad \text { at } Y=0, \\
\frac{\partial F}{\partial Y}=1, \quad H=0, \quad S=0 \quad \text { as } Y \longrightarrow \infty .
\end{gathered}
$$

\section{Results and Discussions}

The system of (14) is solved numerically using the Kellerbox scheme as been applied by Cebeci $[14,15]$ and Ali et al. [18]. The numerical results obtained include the skin friction coefficient, the Nusselt number and the separation time for 
TABLE 3: The separation times along the cylinder surface when $\operatorname{Pr}=7$ and $\alpha=1.25$ (assisting flow).

\begin{tabular}{lccccccc}
\hline$x$ & $K=0$ & $n=0$ & $n=1 / 2$ & $n=0$ & $n=1 / 2$ & $n=0$ \\
\hline $180^{\circ}$ & 1.1033 & 0.8936 & 0.9649 & 0.8040 & 0.9122 & 0.7591 \\
$171^{\circ}$ & 1.1285 & 0.9106 & 0.9839 & 0.8185 & 0.9292 & 0.7726 \\
$162^{\circ}$ & 1.2112 & 0.9654 & 1.0466 & 0.8652 & 0.9847 & 0.8158 \\
$153^{\circ}$ & 1.3798 & 1.0727 & 1.1701 & 0.9555 & 1.0928 & 0.8988 \\
$144^{\circ}$ & 1.7204 & 1.2689 & 1.4014 & 1.1182 & 1.2896 & 1.0463 \\
$135^{\circ}$ & - & 1.6672 & 1.9075 & 1.4302 & 1.6857 & 1.0466 \\
$126^{\circ}$ & - & - & - & 2.2452 & - & 1.2238 \\
\hline
\end{tabular}

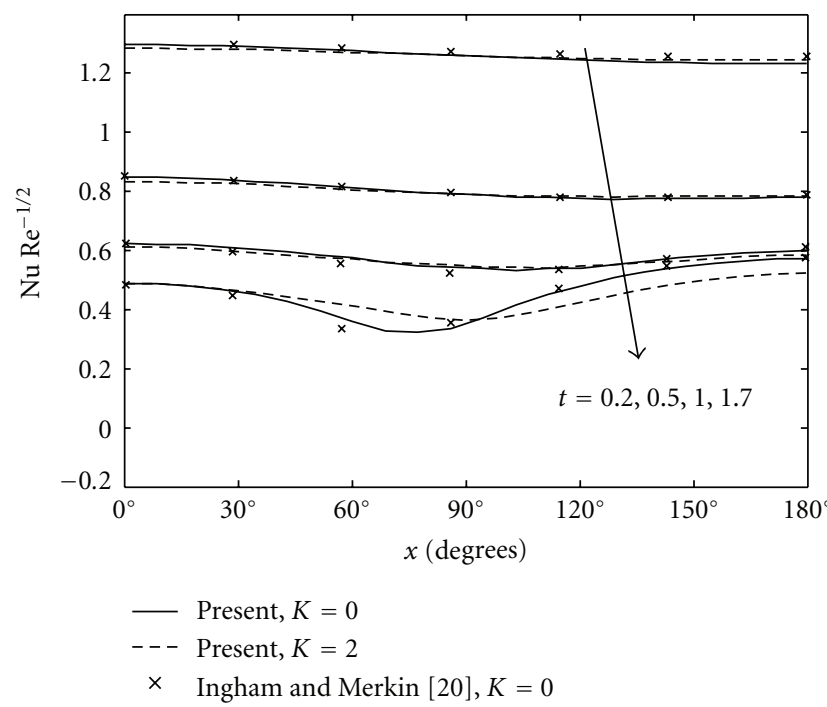

Figure 3: Variation of the Nusselt number with $x$ for $K=0,2$ and for various values of $t$ when $\operatorname{Pr}=1, \alpha=-3$ (opposing flow) and $n=0$.

all points along the cylinder surface. Table 1 presents the separation times around a cylinder surface for some values of $K$ when $\operatorname{Pr}=1$ and $\alpha=-3$ (opposing flow). It can be seen that separation occurs everywhere along the cylinder surface. Also, the separation times increase with the increasing values of $K$. For each value of $K(K \neq 0)$ and at a given $x$ location, the separation time is higher for the case of $n=1 / 2$ (weak concentration) than $n=0$ (strong concentration).

A similar pattern can be observed in the case when $\operatorname{Pr}=7$ and $\alpha=-3$ (opposing flow) is considered (see Table 2). We observed that on a given $x$ location, the separation time for the case of $\operatorname{Pr}=7$ is higher compared to the case of $\operatorname{Pr}=$ 1 , which is in agreement with the results reported by Takao [13]. There are some locations where flow do not separate for the case of $\operatorname{Pr}=7$. We have also found that $K=0$ leads to an earlier location of separation compared to the case of $K \neq 0$.

Table 3 shows the separation time along the cylinder surface when $\operatorname{Pr}=7$ and $\alpha=1.25$ (assisting case). The opposite trend can be seen here where the separation times decrease with increasing values of $K$ since it is known that the micropolar fluids show drag reduction compared to

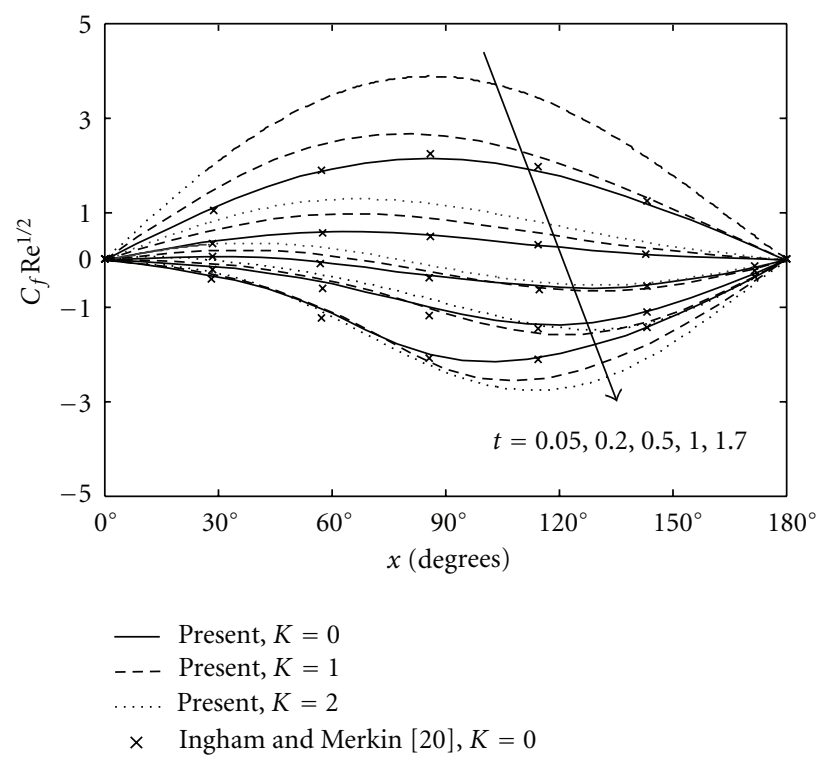

FIGURE 4: Variation of the skin friction coefficient with $x$ for $K=$ 0,1 and 2 with various values of $t$ when $\operatorname{Pr}=1, \alpha=-3$ (opposing flow) and $n=0$.

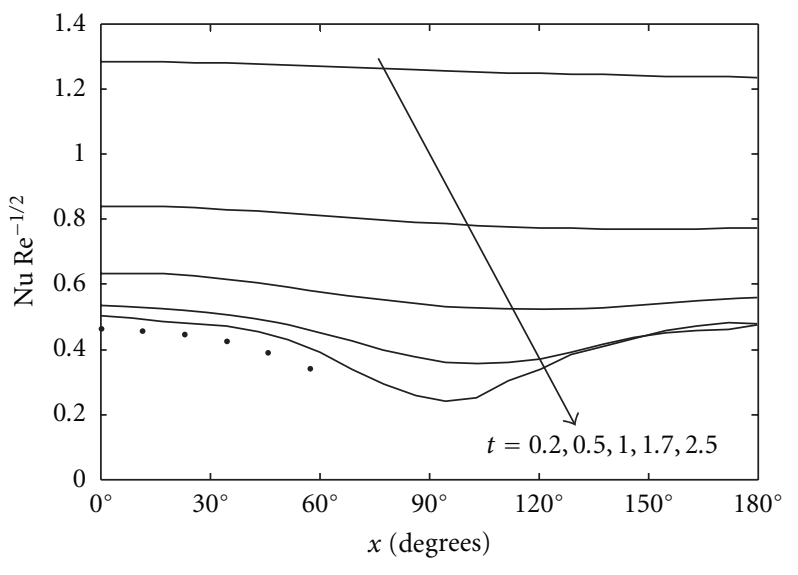

- Steady state

FIGURE 5: Variation of the Nusselt number with $x$ for $K=2$ and for various values of $t$ when $\operatorname{Pr}=1, \alpha=-1$ (opposing flow) and $n=1 / 2$. 


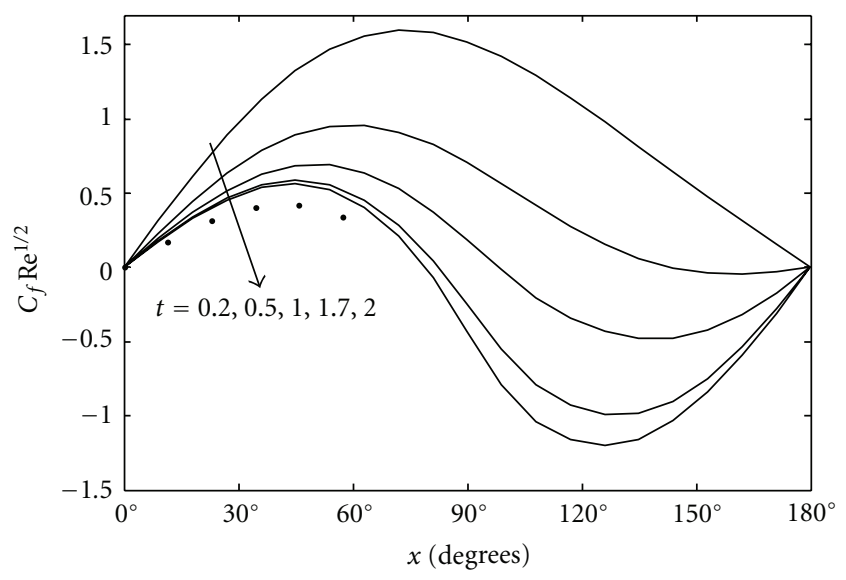

- Steady state

FIGURE 6: Variation of the skin friction coefficient with $x$ for $K=2$ and for various values of $t$ when $\operatorname{Pr}=1, \alpha=-1$ (opposing flow) and $n=1 / 2$.

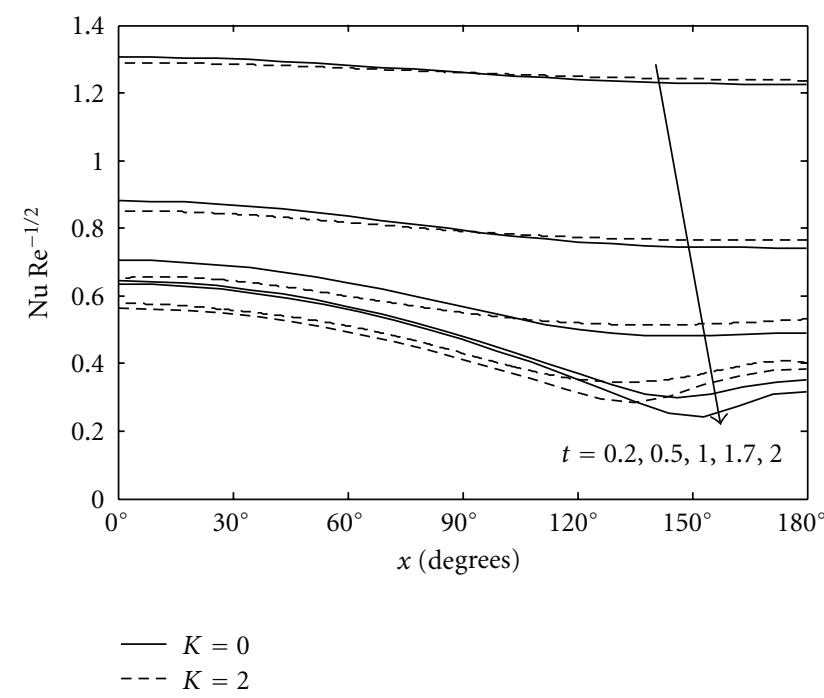

Figure 7: Variation of the Nusselt number with $x$ for $K=0,2$ and for various values of $t$ when $\operatorname{Pr}=1, \alpha=1.25$ (assisting flow) and $n=0$.

Newtonian fluids, see also Cheng and Wang [33]. It can also be seen that as $t$ increases, the separation point moves from the rear stagnation point up to $x=144^{\circ}$ when $K=0$. The case of $K \neq 0$ could move the separation point forward.

Figures 2(a) and 2(b) show the variation of the separation time $t_{s}$ of the boundary layer near both the forward stagnation point $\left(x=0^{\circ}\right)$ and rear stagnation point $(x=$ $180^{\circ}$ ) of the cylinder. Here, the mixed convection parameter $\alpha$ are considered for the range of $-5 \leq \alpha<2$ while the material parameter, $K$ are taken as $K=0$ (Newtonian fluid) and $K=2$ (micropolar fluid) with $n=0$ (strong concentration) and $n=1 / 2$ (weak concentration). For validation purpose, we compare our results with the results reported by Ingham and Merkin [20] for $K=0$, and we notice a good agreement between these results. Near the

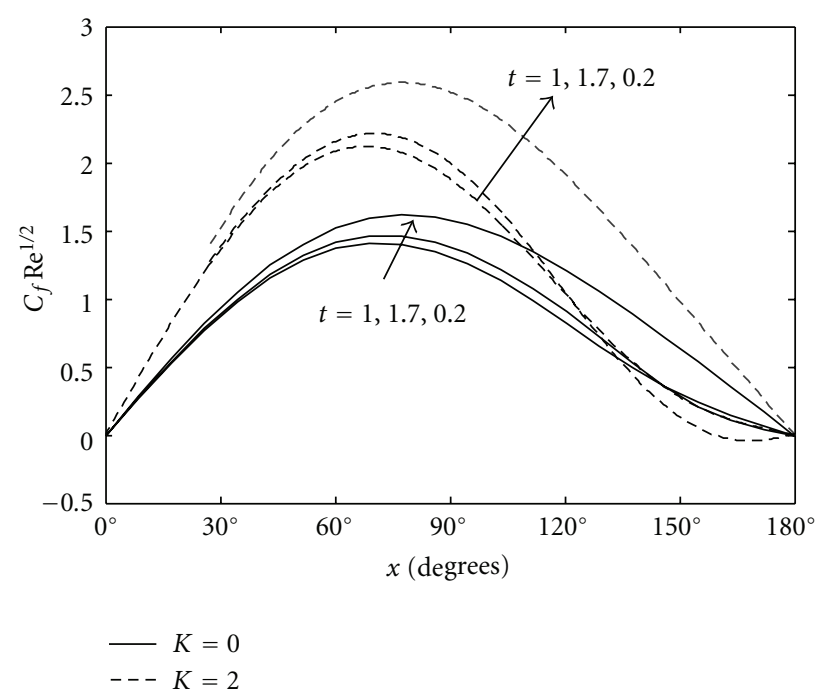

FIGURE 8: Variation of the skin friction coefficient with $x$ for $K=$ 0,2 and for various values of $t$ when $\operatorname{Pr}=1, \alpha=1.25$ (assisting flow) and $n=0$.

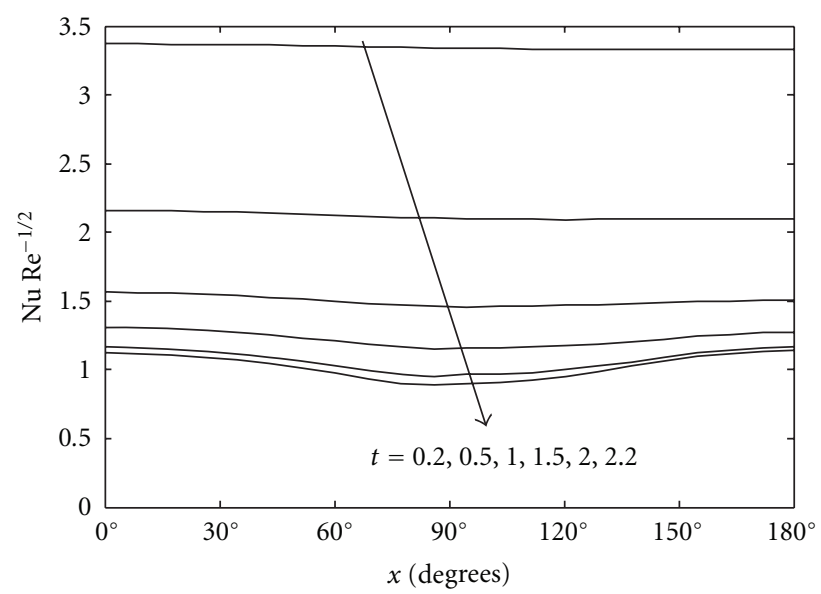

FIGURE 9: Variation of the Nusselt number with $x$ for $K=2$ and for various values of $t$ when $\operatorname{Pr}=7, \alpha=-3$ (opposing flow) and $n=0$.

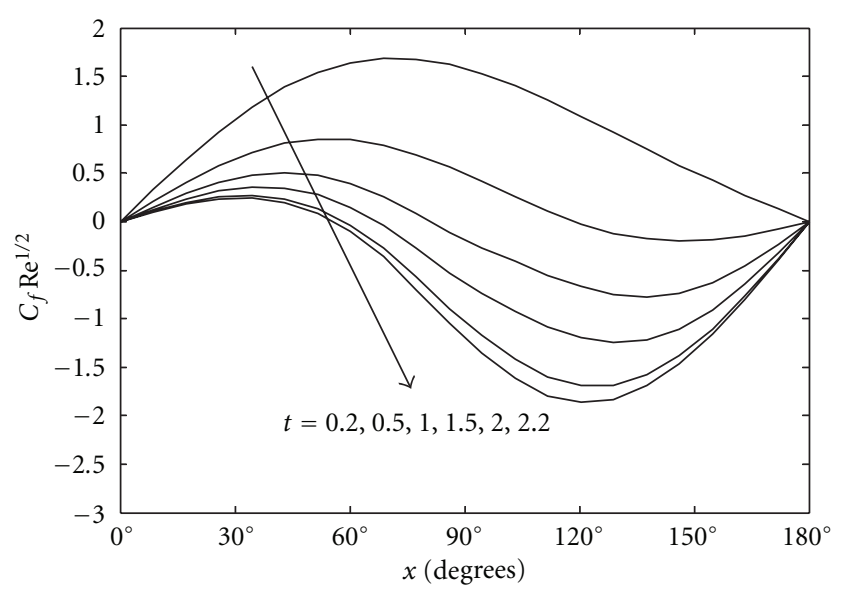

FIgURE 10: Variation of the skin friction coefficient with $x$ for $K=2$ and for various values of $t$ when $\operatorname{Pr}=7, \alpha=-3$ (opposing flow) and $n=0$. 


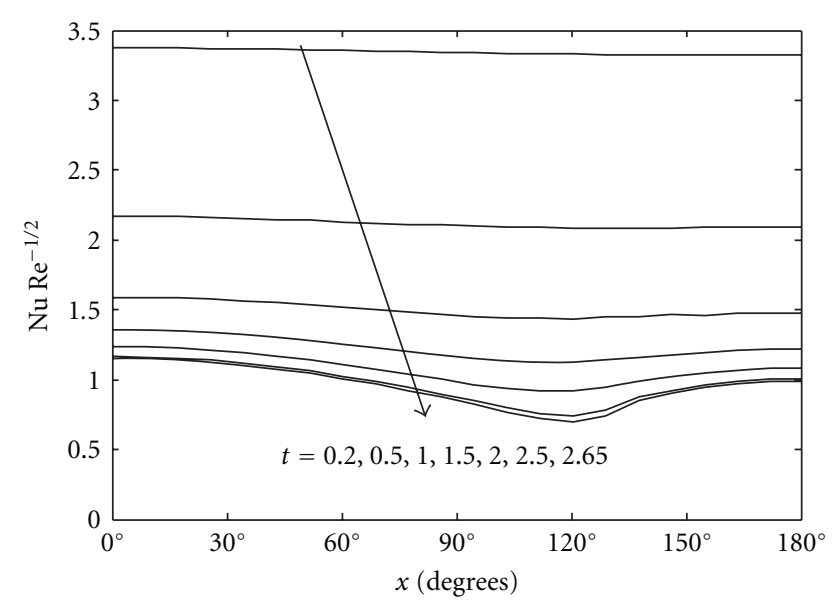

Figure 11: Variation of the Nusselt number with $x$ for $K=2$ and for various values of $t$ when $\operatorname{Pr}=7, \alpha=1.25$ (assisting flow) and $n=0$.

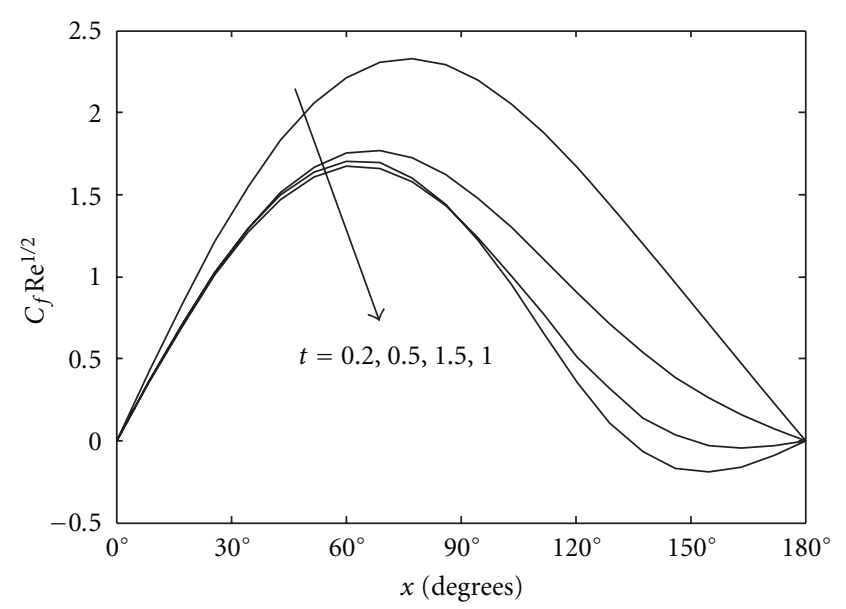

FIgURE 12: Variation of the skin friction coefficient with $x$ for $K=$ 2 and for various values of $t$ when $\operatorname{Pr}=7, \alpha=1.25$ (assisting flow) and $n=0$.

forward stagnation point, it can be seen that the separation time for a micropolar fluid is delayed when compared to the Newtonian fluid for the case of opposing flow $(\alpha<0)$. In contrast, no separation is detected for assisting flow $(\alpha>0)$. Near the rear stagnation point of the cylinder, the separation time for micropolar fluid problem is also a bit delayed for the case of opposing flow $(\alpha<0)$. On the other hand, it can be noticed that for both cases of forced convection $(\alpha=0)$ and assisting flow $(\alpha>0)$, the separation takes place earlier for a micropolar fluid when compared to a Newtonian fluid. Moreover, the separation time near the forward and near the rear stagnation points for the material parameter $K=2$ with the parameter $n=1 / 2$ which is higher if compared to those with the parameter $n=0$.

Figures 3 and 4 show the variation of the Nusselt number $\mathrm{NuRe}^{-1 / 2}$ and skin friction $C_{f} \mathrm{Re}^{1 / 2}$ with the position $x$ along the cylinder for $\alpha=-3$ (opposing case) for $K=0$ (Newtonian fluid) and $K=2$ (micropolar fluids). It is noted that our results when $K=0$ are well agreed with the results obtained by Ingham and Merkin [20]. The results for the micropolar fluids when $K=2$ and $n=0$ are also presented in these figures. When compared to the Newtonian case for the small time solution $(t \leq 1)$, the Nusselt number for micropolar case is lower near the forward stagnation point of the cylinder $(x=0)$. As $t$ increases, different behavior can be seen where the Nusselt number for micropolar case is lower near the rear stagnation point of the cylinder. In addition, the skin friction for micropolar fluid case $(K \neq 0)$ is higher compared to the skin friction of Newtonian fluid $(K=0)$ up to $t=0.2$. From the skin friction value at $t=0.5$, it can be seen that the zero skin friction on the $x$-station is delayed in the micropolar fluid case. Therefore, it can be concluded that micropolar fluid can delay the flow separation for the case of opposing mixed convection past a cylinder.

The variation of the Nusselt number and skin friction coefficient with $x$ for the case of micropolar fluid when $K=2, n=0.5$ and $\alpha=-1$ (opposing case) are shown in Figures 5 and 6 . The steady state results obtained by Nazar et al. [21] are also included in these figures. In Figures 7 and 8, we show the variation of the Nusselt number and the skin friction with $x$ for $\alpha=1.25$ (assisting case) and several values of time $t$ when $K=0$ and $K=2$ with $n=0$. It can seen from Figure 7 that near the lower stagnation point of the cylinder, the Nusselt number are lower for a micropolar fluid than for a Newtonian fluid for all values of $t$ considered. On the other hand, this value is higher for micropolar fluid when it reaches the rear stagnation point. The steady-state solution can be observed as $t$ increases.

Figures 9 and 10 show the variation of Nusselt number and the skin friction along the surface of the cylinder for various values of $t$ when $K=2, \operatorname{Pr}=7$ and $\alpha=-3$ (opposing flow). This variation for Nusselt number and skin friction when $\alpha=1.25$ (assisting flow) are shown in Figures 11 and 12. It is observed that the Nusselt number and skin friction coefficient are higher for the case of $\operatorname{Pr}=7$ compared to the case of $\operatorname{Pr}=1$. The location of separation in the $x$ station is delayed when the case of $\operatorname{Pr}=7$ is considered. In addition, increasing the mixed convection parameter $\alpha$ will also lead to an increase of Nusselt number and skin friction coefficient along the cylinder surface.

\section{Nomenclature}

$\begin{array}{ll}a: & \text { Radius of the cylinder } \\ C_{f}: & \text { Skin friction coefficient } \\ f \text { or } F: & \text { Reduced stream function } \\ \mathrm{Gr}: & \text { Grashof number } \\ h \text { or } H: & \text { Reduced microrotation } \\ j: & \text { Microinertia density } \\ K: & \text { Micropolar material parameter } \\ n: & \text { Ratio of the microrotation vector component } \\ & \text { to the fluid skin friction at the wall } \\ N: & \text { Nondimensional component of the } \\ & \text { microrotation vector normal to } x y \text {-plane } \\ \text { Nu: } & \text { Nusselt number } \\ \operatorname{Pr}: & \text { Prandtl number } \\ q_{w}: & \text { Convection heat transfer coefficient }\end{array}$


Re: Reynolds number

$s$ or $S$ : Reduced temperature

$\bar{t}$ : $\quad$ Dimensional time

$t$ : Non-dimensional time

$t_{s}$ : $\quad$ Separation time

$\bar{T}: \quad$ Dimensional fluid temperature

$T$ : Non-dimensional fluid temperature

$T_{\infty}$ : External temperature

$T_{w}$ : $\quad$ Surface temperature

$\bar{u}, \bar{v}: \quad$ Dimensional velocity components along $\bar{x}$ and $\bar{y}$ axes

$u, v$ : Non-dimensional velocity components along $x$ and $y$ axes

$\bar{u}_{e}(\bar{x})$ : Dimensional external velocity

$U_{\infty}$ : Reference velocity

$\bar{x}, \bar{y}: \quad$ Dimensional Cartesian coordinates measured along the surface of the cylinder and normal to it, respectively

$x, y$ : Non-dimensional Cartesian coordinates measured along the surface of the cylinder and normal to it, respectively.

\section{Greek Symbols}

$\alpha$ : Mixed convection parameter

$\beta$ : Thermal expansion coefficient

$\gamma$ : Spin gradient viscosity

$\eta$ : Similarity variable

$\kappa:$ Vortex viscosity

$\mu$ : Dynamic viscosity

$v$ : Kinematic viscosity

$\rho:$ Density

$\tau_{w}:$ Wall shear stress

$\psi$ : Stream function.

\section{Acknowledgment}

The authors are indebted to the referee for his valuable comments and suggestions, which lead to the improvement of the paper.

\section{References}

[1] W. J. McCroskey, "Some current research in unsteady fluid dynamics. The 1976 Freeman scholar lecture," Journal of Fluids Engineering, vol. 99, no. 1, pp. 8-39, 1977.

[2] K. Stewartson, "The theory of unsteady laminar boundary layers," in Advances in Applied Mechanics, vol. 6, pp. 1-37, Academic Press, New York, NY, USA, 1960.

[3] J. Stuart, "Unsteady boundary layers," in Laminar Boundary Layers, L. Rosenhead, Ed., pp. 349-406, Clarendon Press, Oxford, UK, 1964.

[4] N. Riley, "Unsteady laminar boundary layers," SIAM Review, vol. 17, no. 2, pp. 274-297, 1975.

[5] N. Riley, "Unsteady viscous flows," Scientific Progress, vol. 74, pp. 361-377, 1990.

[6] D. P. Telionis, Unsteady Viscous Flows, Springer, Berlin, Germany, 1981.

[7] I. Pop, D. B. Ingham, and J. H. Merkin, "Transient convecive heat transfer in external flow," in Time-Dependent Nonlinear
Convection, P. A. Tyvand, Ed., vol. 19 of Advances in Fluid Mechanics, pp. 83-114, Computational Mechanics Publications, Southampton, UK, 1998.

[8] W. M. Collins and S. C. R. Dennis, "The initial flow past an impulsively started circular cylinder," Quarterly Journal of Mechanics and Applied Mathematics, vol. 26, no. 1, pp. 53-75, 1973.

[9] W. M. Collins and S. C. R. Dennis, "Flow past an impulsively started circular cylinder," Journal of Fluid Mechanics, vol. 60, no. 1, pp. 21-1973, 1973.

[10] D. P. Telionis and D. Th Tsahalis, "Unsteady laminar separation over impulsively moved cylinders," Acta Astronautica, vol. 1, no. 11-12, pp. 1487-1505, 1974.

[11] M. Bar-Lev and H. T. Yang, "Initial flow field over an impulsively started circular cylinder," Journal of Fluid Mechanics, vol. 72, no. 4, pp. 625-647, 1975.

[12] V. A. Patel, "Time-dependent solutions of the viscous incompressible flow past a circular cylinder by the method of series truncation," Computers \& Fluids, vol. 4, no. 1, pp. 13-27, 1976.

[13] S. Takao, "Short-time solution for unsteady forced convection heat transfer from an impulsively started circular cylinder," International Journal of Heat and Mass Transfer, vol. 21, no. 12, pp. 1505-1516, 1978.

[14] T. Cebeci, "Heat transfer from a circular cylinder impulsively started from rest," Numerical Heat Transfer, Part A, vol. 1, no. 4, pp. 557-567, 1978.

[15] T. Cebeci, "The laminar boundary layer on a circular cylinder started impulsively from rest," Journal of Computational Physics, vol. 31, no. 2, pp. 153-172, 1979.

[16] D. B. Ingham, "Unsteady separation," Journal of Computational Physics, vol. 53, no. 1, pp. 90-99, 1984.

[17] S. Nam, "Higher-order boundary-layer solution for unsteady motion of a circular cylinder," Journal of Fluid Mechanics, vol. 214, pp. 89-110, 1990.

[18] A. Ali, N. Amin, and I. Pop, "The unsteady boundary layer flow past a circular cylinder in micropolar fluids," International Journal of Numerical Methods for Heat and Fluid Flow, vol. 17, no. 7, pp. 692-714, 2007.

[19] J. H. Merkin, "Mixed convection from a horizontal circular cylinder," International Journal of Heat and Mass Transfer, vol. 20, no. 1, pp. 73-77, 1977.

[20] D. B. Ingham and J. H. Merkin, "Unsteady mixed convection from an isothermal circular cylinder," Acta Mechanica, vol. 38, no. 1-2, pp. 55-69, 1981.

[21] R. Nazar, N. Amin, and I. Pop, "Mixed convection boundarylayer flow from a horizontal circular cylinder in micropolar fluids: case of constant wall temperature," International Journal of Numerical Methods for Heat \& Fluid Flow, vol. 13, no. 1, pp. 86-109, 2003.

[22] A. C. Eringen, "Theory of micropolar fluids," Journal of Mathematics and Mechanics, vol. 16, pp. 1-18, 1966.

[23] T. Ariman, M. A. Turk, and N. D. Sylvester, "Microcontinuum fluid mechanics - a review," International Journal of Engineering Science, vol. 11, no. 8, pp. 905-930, 1973.

[24] T. Ariman, M. A. Turk, and N. D. Sylvester, "Applications of microcontinuum fluid mechanics," International Journal of Engineering Science, vol. 12, no. 4, pp. 273-293, 1974.

[25] A. C. Eringen, Microcontinuum Field Theories. II: Fluent Media, Springer, New York, NY, USA, 2001.

[26] G. Łukaszewicz, Micropolar Fluids: Theory and Application, Birkhauser, Basel, Switzerland, 1999.

[27] V. L. Kolpashchikov, N. P. Migun, and P. P. Prokhorenko, "Experimental determination of material micropolar fluid 
constants," International Journal of Engineering Science, vol. 21, no. 4, pp. 405-411, 1983.

[28] M. Kumari and G. Nath, "Unsteady incompressible boundary layer flow of a micropolar fluid at a stagnation point," International Journal of Engineering Science, vol. 22, no. 6, pp. 755-768, 1984.

[29] R. S. Agarwal, R. Bhargava, and A. V. S. Balaji, "Finite element solution of nonsteady three-dimensional micropolar fluid flow at a stagnation-point," International Journal of Engineering Science, vol. 28, no. 8, pp. 851-857, 1990.

[30] G. Ahmadi, "Self-similar solution of imcompressible micropolar boundary layer flow over a semi-infinite plate," International Journal of Engineering Science, vol. 14, no. 7, pp. 639646, 1976.

[31] G. S. Guram and A. C. Smith, "Stagnation flows of micropolar fluids with strong and weak interactions," Computers \& Mathematics with Applications, vol. 6, no. 2, pp. 213-233, 1980.

[32] S. K. Jena and M. N. Mathur, "Similarity solutions for laminar free convection flow of a thermomicropolar fluid past a non-isothermal vertical flat plate," International Journal of Engineering Science, vol. 19, no. 11, pp. 1431-1439, 1981.

[33] C.-Y. Cheng and C.-C. Wang, "Forced convection in micropolar fluid flow over a wavy surface," Numerical Heat Transfer, Part A, vol. 37, no. 3, pp. 271-287, 2000. 

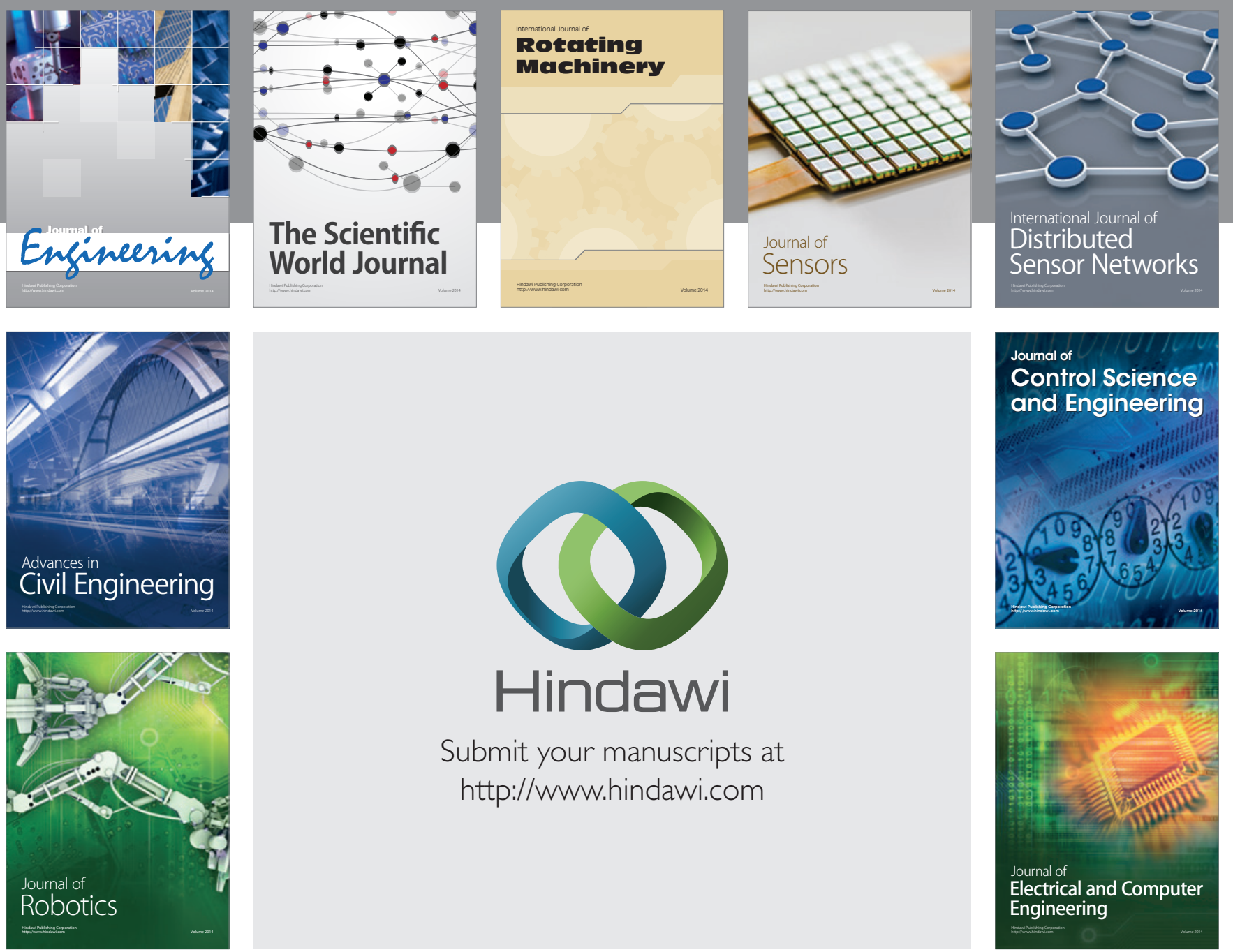

Submit your manuscripts at

http://www.hindawi.com
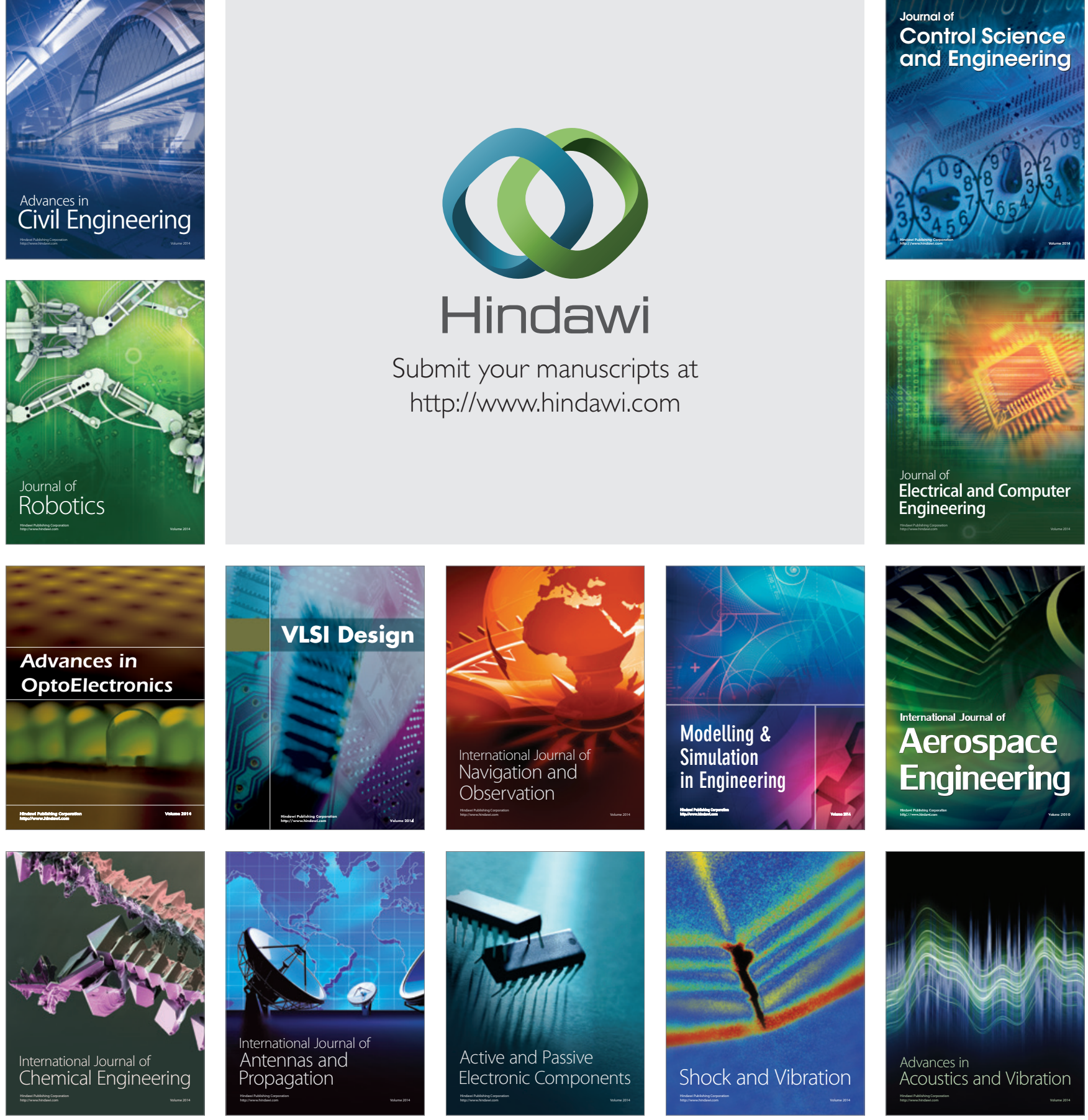